\title{
Correlation of Sperm DNA Fragmentation Index With Seminal Plasma Biochemical Index and Semen Parameters in Infertile Men
}

\section{Xiao-Dong Mao}

Nanjing University of Chinese Medicine

Xinyuan Yang ( $\nabla$ njfyly@sohu.com )

xian jiaotong university

\section{Feng Pan}

Nanjing Medical University

Kang-Sheng Liu

xian jiaotong university

\section{Research Article}

Keywords: DNA fragmentation index, Seminal plasma biochemical, Semen Parameters, Malondialdehyde, Total antioxidant capacity, Fru, infertility

Posted Date: December 15th, 2020

DOl: https://doi.org/10.21203/rs.3.rs-122500/v1

License: (c) (i) This work is licensed under a Creative Commons Attribution 4.0 International License. Read Full License 


\section{Abstract}

In general, routine semen analysis makes only limited predictions about a man's reproductive potential and is not always able to explain why he is infertile. In fact, many male infertility cases are caused by sperm DNA defects, which routine semen quality analyses fail to detect. The relationship between sperm DFI, sperm parameters and their diagnostic value were analyzed and evaluated by observing the seminal parameters of infertile patients without accessory gonadal infection. Specimens of 151 cases were collected from infertile patients who visited the male department of STD and reproductive specialty clinic of our hospital from August 2018 to September 2019. SCD test was performed to measure the DNA fragmentation in native. The routine semen analysis was performed with a semen quality detection system (CFT-920, Jiangsu Ruiqi Life Science \& Tech Dev .Co.Ltd) and supporting reagents. Seminal plasma malondialdehyde (MDA), and total antioxidant capacity (TAC) were assessed. Fructose(Fru) $\square a-$ glucosidase (a-glu), and zinc (Zn) levels are quantitatively detected by kehua-310, a fully automated biochemical tester provided by Nanjing Xindibio. According to DFI level, there were 31 cases in group I (DFI $\leq 15 \%), 81$ cases in group II $(15 \%<\mathrm{DFI}<30 \%)$, and 39 cases in group III (DFI $\geq 30 \%)$. Compared with group II, there were significant differences in sperm survival rate, PR\% and Fru by non-parametric test $(Z=$ -2.16 -2. 43. -2 . 20 ,respectively, $P<0$. 05). There were significant differences in sperm survival rate and PR\% between group I and group III $(t=4.32,4.25$, respectively, $P<0.01)$. Compared with group III, there were significant differences in sperm survival rate and PR\% by non-parametric test $(Z=-3.26,-3.50$, respectively).Sperm DFI was negatively correlated with sperm survival rate and $\mathrm{PR} \%(R=-0.56,-0.46, P<$ $0.01)$. DFI was positively correlated with MDA content $(R=0.42, P<0.01)$ and negatively correlated with TAC $(r=-0.40, P<0.01)$. There was no correlation with age ,abstinence days, semen volume, sperm concentration, percentage of normal form sperm, Fru, a-Glu, Zn $(R=0.15,0.05,0.03,-0.03,-0.2,-0.16$,$0.20,0.03,0.15, p>0.05)$. The survival rate and PR\% of sperm decreased significantly with the increase of DFI level, antioxidant can decrease the rate of DNA fragmentation, antioxidant can decrease the rate of DNA fragmentation. Semen volume and sperm concentration were mainly related to the secretion volume of accessory gonads and total sperm count, but no correlation was found between them and DFI.

\section{Introduction}

Male infertility, a rising concern in reproductive medicine, is caused by a wide variety of spermatogenic abnormalities, including sperm concentration, motility, morphology, and so on. Up to $50 \%$ of infertility is male-factor, which ratio was only $30 \%$ in $2013^{[1]}$. Nowadays, DNA fragmentation index (DFI) has been becoming an adjunct test for the standard semen analysis because lower rates of conception always accompany with the elevated sperm DFI and up to $8 \%$ of infertile men with normal semen analysis results may have a high abnormal sperm $\mathrm{DF}^{[2-3]}$. The damaged sperm DNA may lead to unsuccessful pregnancy outcomes which could not be evaluated by the routine semen analysis. Thus, the sperm DFI has been introduced into the clinical laboratory to diagnose the male infertility ${ }^{[4]}$. The simultaneous detection of the above-mentioned semen parameters can provide a comprehensive understanding of the patient's semen status, sperm motility, sperm morphology, defect degree of genetic material in sperm 
head nucleus and accessory gonadal secretion function, which has important guiding significance for the diagnosis and treatment of infertility.

In this paper, the relationship and diagnostic value between sperm DFI and various sperm parameters were analyzed and evaluated by observing the semen parameters of infertile patients without accessory gonadal infection.

\section{Materials And Methods}

\section{The general information}

The selected 151 infertile patients (ages from 22 to 53 years, mean age $31.6 \pm 3.2$ years)who visited at the male department and reproductive Medicine Center at our hospital in August 2018 to September 2019 were included in the present study. Excluded were those with family diseases, genital trauma and organic lesions, urological and reproductive diseases (like varicocele, cryptorchidism, prostatitis, epididymitis, leukocytopenia, hematospermia and azoospermia) .

Corresponding(blood routine, urine routine, reproductive hormone, serum anti-sperm antibody) tests were normal. Semen test( mycoplasma, chlamydia, gonococcal) was negative.

\section{Ethics}

The research was approved by the Ethics Committee of Women's Hospital of Nanjing Medical University, and was conducted in accordance with the Declaration of Helsinki. An information sheet was provided to all participants. Written informed consent was obtained from all participants. The relevant guidelines and regulations of the local institute were strictly followed when conducting the study. Participants were informed that they could withdraw from the trial without giving a reason.

\section{Semen collection}

Semen samples were collected by masturbation after 2 to 5 days of ejaculatory abstinence (WHO, 2010)

[5]. The duration of abstinence was recorded. Each semen sample was directed into a sterile plastic cup, liquefied in an incubator at $37^{\circ} \mathrm{C}$. After the semen was completely liquefied, at least $2.6 \mathrm{ml}$ of semen was taken for the test $(1.5 \mathrm{ml}$ of the semen was used for routine semen analysis, the left for DNA fragmentation analysis and oxidative stress parameters)..

\section{Rutine semen analysis}

According to Laboratory Manual of the WHO for the Examination and Processing of Human Semen (5th edition ) and WHO Manual for the Standardized Investigation, Diagnosis and Management of the Infertile Male ${ }^{[5]}$, the routine semen analysis was performed with a semen quality detection system (CFT-920, Jiangsu Ruiqi Life Science \& Tech Dev .Co.Ltd) and supporting reagents. Main parameters were as 
follows $^{[6]}$. Image acquisition frame: low and middle sperm concentration collected at $20 \mathrm{~Hz}$, and high sperm concentration at $7 \mathrm{~Hz}$; acquisition interval: $3 \mathrm{~ms}$; maximum sperm motile velocity: $200 \mathrm{um} \mathrm{s}^{-1}$; area range of spermatozoa head detected at 7-60 um². Index of sperm motility: straight line velocity (VSL). Grayscale thresholds were set to collect spermatozoa and exclude nonsperm granules. According to the thresholds set for sperm analysis, sperm images were collected and analyzed.

\section{Normal Parameters}

(1) Semen volume $\geq 1.5 \mathrm{ml} ; \mathrm{pH} \geq 7.2$; Liquefaction time $<60 \mathrm{~min}$; Total motile spermatozoa $\geq 39 \times$ $10^{6} /$ an entire ejaculate; Sperm count $\geq 15 \times 10^{6} / \mathrm{ml}$; Motility $\geq 40 \%$; Vitality $\geq 50 \%$; Morphology: Proportion of sperms with normal morphology $\geq 4 \%$.

(2) Sperm with normal forward movement: Sperms of grade $(A+B) \geq 32 \%$, or sperms of grade $A \geq 25 \%$, 10]

\section{Diagnostic criteria of asthenozoospermia}

Mild: $30-50 \%$ sperms at grade $(A+B)$, or $10-25 \%$ sperms at grade $A$.

Moderate: $30-50 \%$ sperms at grade $(A+B)$, and sperms of grade $A \leq 10 \%$.

Severe: Sperms of grade $(B+C) \leq 30 \%$, and absence of grade $A$ sperms ${ }^{[7,10]}$.

\section{Sperm morphology assessment}

For morphological evaluations, seminal smears were stained with Diff-Quik (MICROPTIC S.L. Co., Barcelona, Spain $)^{[8]}$. Approximately $10 \mu$ of sperm was smeared into a thin and homogeneous layer on a clean glass slide and was air-dried at room temperature for at least $10 \mathrm{~min}$. The slides were stained and observed under a brightfield microscope (BH-2; Olympus, Tokyo, Japan) at $1000 \times$ magnification. According to WHO guidelines, a sperm with deformed head, or midpiece, or principal piece was counted as. SDI (sperm deformity index) = number of deformed sperm/number of total sperm. For each semen sample, at least 200 sperms (or the whole sperm if the slide had less than 200 sperm) were counted via a double-blinded method. Then, the percentage of sperm with normal morphology was calculated ${ }^{[9]}$.

\section{$\operatorname{DFI}($ SCD test $)$}

Using the SpermFunc ${ }^{\mathrm{TM}}$ DNAf kit (BRED Life Science, Shenzhen, China), SCD test was performed to measure the DNA fragmentation in native and DGC-separated semen. Gelled aliquots of low-melting-point agarose in the kit were provided for semen sample processing in Eppendorf tubes. Eppendorf tubes were placed in a water bath at $80{ }^{\circ} \mathrm{C}$ for $20 \mathrm{~min}$ to melt the agarose and then transferred to a water bath at $37^{\circ} \mathrm{C}$ for $5 \mathrm{~min}$ for temperature equilibration. A total of $60 \mu \mathrm{L}$ of sampled semen was added to and mixed with the agarose in the Eppendorf tubes. Then, $30 \mu \mathrm{L}$ of semen-agarose mixture was pipetted onto precoated slides in the kit that were covered with a $22 \times 22-\mathrm{mm}$ coverslip. The slides were placed on a cold plate in the refrigerator $\left(4^{\circ} \mathrm{C}\right)$ for $5 \mathrm{~min}$, allowing the agarose to produce microgel in which the sperm 
cells were embedded. The coverslips were gently removed, and the slides were immediately immersed horizontally in solution A and incubated for $7 \mathrm{~min}$. Next, the slides were horizontally immersed in solution $\mathrm{B}$ for $25 \mathrm{~min}$. After being washed for $5 \mathrm{~min}$ in a tray with abundant distilled water, the slides were dehydrated in gradient concentrations of ethanol (70\%, $90 \%, 100 \%$; respectively) for 2 min, air-dried, and stored at room temperature in opaque closed boxes ${ }^{[10]}$.

For bright-field microscopy, the slides were horizontally covered with a mixture of Wright's staining solution (BRED Life Science, Shenzhen, China) and phosphate buffer solution (BRED Life Science, Shenzhen, China) (1:2) for 15 min with continuous airflow. Then, the slides were washed in running water for $10 \mathrm{~s}$ and allowed to dry. Strong staining was recommended to allow the periphery of the dispersed DNA loop halos more visible. A minimum of 500 sperm were counted on each sample under the $100 \times$ magnification ${ }^{[11]}$.

Normal spermatic DNA presented radiate halos and damaged spermatic DNA presented no or small halos. Fragmented sperm referred to those having a small or no halo. The thickness of the halo on one side was less than the $1 / 3$ diameter of the head's thinnest part ${ }^{[12]}$. The rate of sperm DNA fragmentation $(\%)=$ the number of sperm with fragmented DNA $\div$ the total number of sperm $\times 100 \%$, and $\otimes 25 \%$ was considered normal.

\section{Quantitative detection of seminal plasma biochemical indexes}

Spectrophotometry was used to test the level of TAC $(\mathrm{U} / \mathrm{L})$ and $M D A(\mathrm{nmol} / \mathrm{mL})$. MDA levels were determined using the thiobarbituric acid (TBA) method. Semen samples were centrifuged at $4{ }^{\circ} \mathrm{C}$ for 15 min with a speed of $2000 \mathrm{r} / \mathrm{min}$. The supernatant was mixed with the reagents supplied in an MDA Assay Kit (Nanjing Jiancheng Bioengineering Corporation, China, A003-2) and incubated at $95^{\circ} \mathrm{C}$ for $40 \mathrm{~min}$. Having been cooled at room temperature, the mixture was centrifuged at $4000 \mathrm{~g}$ for $10 \mathrm{~min}$. The absorbance of the supernatant was measured at $530 \mathrm{~nm}$. All operations were performed according to the manufacturer's instructions. The MDA concentrations were expressed as $\mathrm{nmol} / \mathrm{mL}^{[13]}$.

Fructose(Fru), a-glucosidase (,a-glu), and zinc ( $\mathrm{Zn}$ ) levels are quantitatively detected by kehua-310, a fully automated biochemical tester provided by Nanjing Xindibio. Normal reference range of laboratory: Fru 11. $01 \sim 43.07 \mathrm{mmol} / \mathrm{L}$, a -Glu 109. 6-570. $8 \mathrm{mmol} / \mathrm{L}, \mathrm{Zn} 1.09 \sim 4.86 \mathrm{mmol} / \mathrm{L}$, coefficient of variation $<7 \%$.

\section{Statistical analysis}

The software(SPSS 22.0)analyzed the data, and the measurement data description was expressed as $x \pm$ $s$ in accordance with the normal distribution, and the skewed distribution was expressed as $M(P 25$, P75).Independent sample $T$ test was used to test the homogeneity of normal distribution and variance, and mann-Whitney test was used to test skewed distribution. The correlation was analyzed by Pearson, and the correlation coefficient was expressed by $p<0.05$ indicates that the difference is statistically significant. 


\section{Results}

Basic information description of the research object

Table 1 Basic information description of the research object

The quantitative detection of semen parameters, sperm DFI, seminal plasma Fru, a -Glu and Zn in 151 infertility patients was completed. See Table 1.

\begin{tabular}{|c|c|c|}
\hline \\
\hline \multicolumn{3}{|r|}{$\mathrm{M}\left(P_{25^{\square}} P_{75}\right)$} \\
\hline Abstinence days ( $d$ ) & $4 . .2 \pm 1.6$ & 4. $1(3 . .0,7.2)$ \\
\hline age $(y r)$ & $31 . .6 \pm 3.2$ & $30 . .1(27.0, \quad 33.5)$ \\
\hline Sperm concentration $\left(\times 10^{6} / \mathrm{ml}\right)$ & 110. $3 \pm 81.1$ & 85. 3( $41.4,165.3)$ \\
\hline Semen volume ( $\mathrm{ml})$ & $3.5 \pm 1.4$ & $3.5(2.4 \rrbracket 4.4)$ \\
\hline Sperm survival rate( \% ) & $36.2 \pm 13.2$ & $36.2(28.2 \rrbracket 40.8)$ \\
\hline Normal sperm( \% ) & $2.9 \pm 3.4$ & 2. $1(1.0 \otimes 3.0)$ \\
\hline $\mathrm{a}-\mathrm{Glu}(\mathrm{mmol} / \mathrm{L})$ & $491.3 \pm 279.1$ & 427. 3(231. 6ه672. 5) \\
\hline Fru( $\mathrm{mmol} / \mathrm{L})$ & 14. $2 \pm 6.1$ & 11. $2(9.3 \rrbracket 22.6)$ \\
\hline $\mathrm{Zn}(\mathrm{mmol} / \mathrm{L})$ & 3. $0 \pm 1.2$ & 2. $9(1.9 \bowtie 3.9)$ \\
\hline DFI( \%) & $24 \rrbracket 2 \pm 13.5$ & 23. $0(18.0 \unrhd 23.7)$ \\
\hline
\end{tabular}

\section{Comparison of semen parameters between different sperm DFI levels}

Table 2 Comparison of semen parameters between different sperm DFI levels 


\begin{tabular}{|c|c|c|c|c|c|c|}
\hline & & 1 & & $\|$ & & III \\
\hline \multirow[t]{2}{*}{ Parameter } & & $\begin{array}{l}\mathrm{M}(P 25 \rrbracket \\
P 75)\end{array}$ & & $\begin{array}{l}\mathrm{M}(P 25 \rrbracket \\
P 75)\end{array}$ & & $\begin{array}{l}\mathrm{M}(P 25 \rrbracket \\
P 75)\end{array}$ \\
\hline & $x \pm s$ & & $x \pm s$ & & $x \pm s$ & \\
\hline Abstinence days(d) & $\begin{array}{l}4.2 \pm \\
1.8\end{array}$ & $\begin{array}{l}4 \rrbracket 0( \\
3.2 \rrbracket \\
5.6)\end{array}$ & $4.0 \pm 1.9$ & $\begin{array}{l}3.8(3.0 \rrbracket \\
7.2)\end{array}$ & $\begin{array}{l}4 \rrbracket 8 \\
\pm 2 \\
2\end{array}$ & $\begin{array}{l}\text { 4. } 9(3.5 \rrbracket \\
\text { 8. } 0)\end{array}$ \\
\hline $\operatorname{age}(y)$ & $\begin{array}{l}30.7 \pm \\
6.0\end{array}$ & $\begin{array}{l}28 \rrbracket 9(23 . \\
0 \rrbracket 33.0)\end{array}$ & $33.3 \pm 5.8$ & $\begin{array}{l}36.0(25 . \\
0 \otimes 38.2)\end{array}$ & $\begin{array}{l}33.5 \\
\pm 5 .\end{array}$ & $\begin{array}{l}36 \otimes 0(26 . \\
0 \otimes 39.0)\end{array}$ \\
\hline Sperm survival rate( \% ) & $\begin{array}{l}41.3 \pm \\
11.0\end{array}$ & $\begin{array}{l}\text { 40. 2( } 35 . \\
6 \varangle 50.0)\end{array}$ & $37.1 \pm 12.0$ & $\begin{array}{l}35.5(30 . \\
7 \llbracket 44.5)\end{array}$ & $\begin{array}{l}26.8 \\
\pm 15 . \\
1^{\star \star}\end{array}$ & $\begin{array}{l}27.6(18 . \\
5 \rrbracket 38.3) \\
\# \#\end{array}$ \\
\hline Semen volume( ml) & $\begin{array}{l}3.4 \pm \\
1.2\end{array}$ & $\begin{array}{l}\text { 3. } 0(2.3 \square \\
4.1)\end{array}$ & $3.3 \pm 1.0$ & $\begin{array}{l}\text { 3. } 02(2 . . \\
2 \varangle 4.0)\end{array}$ & $\begin{array}{l}3.6 \\
\pm 1 . \\
1\end{array}$ & $\begin{array}{l}3 \rrbracket 6(3 . \\
6 \rrbracket 4.6)\end{array}$ \\
\hline $\begin{array}{l}\text { Sperm } \\
\text { concentration }\left(\times 10^{6} / \mathrm{ml}\right)\end{array}$ & $\begin{array}{l}107 \\
7 \pm 61 \\
7\end{array}$ & $\begin{array}{l}89.2(60.1 \\
, 150.1)\end{array}$ & $105.1 \pm 50.90$ & $\begin{array}{l}95.9(55 . \\
1 \otimes 193.2)\end{array}$ & $\begin{array}{l}105 \\
2 \pm \\
107 \\
7\end{array}$ & $\begin{array}{l}59.3(21 . \\
8 \otimes 170.6)\end{array}$ \\
\hline Normal sperm( \% ) & $\begin{array}{l}2.6 \pm \\
2.6\end{array}$ & $\begin{array}{l}\text { 2. } 0(1.0 \square \\
\text { 3.0) }\end{array}$ & $2.3 \pm 2.0$ & $\begin{array}{l}\text { 2. } 0(1.0 \mathrm{D} \\
4.6)\end{array}$ & $\begin{array}{l}1.7 \\
\pm 1 \\
3\end{array}$ & $\begin{array}{l}1 . .6( \\
0.5,1.6) \#\end{array}$ \\
\hline PR\% & $\begin{array}{l}37.6 \pm \\
11.4\end{array}$ & $\begin{array}{l}36 \rrbracket 0(30 \rrbracket \\
.2 \varangle 41 \rrbracket 2)\end{array}$ & $30.6 \pm 9.5$ & $\begin{array}{l}28 \rrbracket 5(23 . \\
3 \rrbracket 37.1) \\
*\end{array}$ & $\begin{array}{l}21.3 \\
\pm 13 . \\
6^{\star *}\end{array}$ & $\begin{array}{l}20.6(10 . \\
7 \rrbracket 32 . \\
2) \# \#\end{array}$ \\
\hline $\mathrm{Zn}(\mathrm{mmol} / \mathrm{l})$ & $\begin{array}{l}2.5 \pm \\
1.0\end{array}$ & $\begin{array}{l}2.8(1.6 \square \\
3.2)\end{array}$ & $2.5 \pm 1.0$ & $\begin{array}{l}\text { 3. } 0(1 . \\
9,5.0)\end{array}$ & $\begin{array}{l}2.8 \\
\pm 1 . \\
3\end{array}$ & $\begin{array}{l}2 \searrow 9(2.0 \\
, 3.9)\end{array}$ \\
\hline Fru( mmol / I) & $\begin{array}{l}\text { 17. } 3 \pm \\
\text { 7. } 0\end{array}$ & $\begin{array}{l}\text { 16. 22(10. } \\
9,23.1)\end{array}$ & $13.2 \pm 6.5$ & $\begin{array}{l}\text { 12. } 2(9 \\
1,19.6)^{*}\end{array}$ & $\begin{array}{l}12.7 \\
\pm 8 . \\
2^{*}\end{array}$ & $\begin{array}{l}10.3(7 . \\
5 \otimes 19.7)\end{array}$ \\
\hline $\mathrm{a}-\mathrm{Glu}(\mathrm{mmol} / \mathrm{l})$ & $\begin{array}{l}417.2 \\
\pm 2018 \\
2\end{array}$ & $\begin{array}{l}\text { 381. 8( } \\
\text { 271. 3\ } \\
\text { 522. 6) }\end{array}$ & $\begin{array}{l}423.3 \pm \\
218.5\end{array}$ & $\begin{array}{l}\text { 509. 3( } \\
\text { 266. 1区 } \\
\text { 725. 0) }\end{array}$ & $\begin{array}{l}436 . \\
5 \pm \\
306 \\
3\end{array}$ & $\begin{array}{l}\text { 420. 7( } \\
\text { 190. 3区 } \\
733.3)\end{array}$ \\
\hline
\end{tabular}

Compared with Group I, *: $P<0.05 \rrbracket * *: P \rrbracket 0.01$; Compared with Group II,\#: $P \rrbracket 0.05 ; \# \#: P \rrbracket 0.01$.

According to DFI level, there were 31 cases in group I (DFI $\leq 15 \%), 81$ cases in group II (15\%<DFI < 30\%), and 39 cases in group III (DFI $\geq 30 \%)$. Compared with group II, there were significant differences in sperm survival rate, $\mathrm{PR} \%$ and Fru by non-parametric test $(Z=-2.16$. -2. 43. - 2. 201, respectively, $P<0$. 05). There were no significant differences in age, abstinence days, semen volume, sperm concentration, percentage of normal sperm, a-Glu and $Z n(Z=-1.31,-0.71,-0.09,-0.62,-0.53,-1.31,-1.90$,respectively. $P>0.05)$. 
There were significant differences in sperm survival rate and PR\% between group I and group III $(t=4.32$, 4. 25, respectively, $P<0$. 01). There was a significant difference in Fru $(t=2.30, P<0.05)$; There was no significant difference between semen volume and $Z n$ by t-test $(t=-0.93 .-1.50$, respectively, $P>0$. 05); There were no significant differences in age, abstinence days, sperm concentration, percentage of normal sperm and Glu between the two groups by non-parametric test $(Z=-1.52 .-0.63,-1.06,-1$. 56. -0 . $013, P>0.05)$. Compared with group III, there were significant differences in sperm survival rate and PR\% by non-parametric test $(Z=-3.26 .-3.50$, respectively.$P$ number test showed significant difference $(Z=$ $-3.41,-3.29$, respectively, $P<0.01)$; There were significant differences in the percentage of normal sperm $(Z=-2.30, P<0.05)$; There were no significant differences in age, abstinence days, semen volume, sperm concentration, Fru, alpha-Glu, and Zn ( $Z$ was -0 . 60, - - 32, -1. 69, -1. 53, -0. 58, -1. 20, -0. 18, respectively .p >0. 05, see Table 2 .

\section{Correlation analysis between sperm DFI and semen parameters}

Table3 Correlation analysis between sperm DFI and semen parameters

\begin{tabular}{|c|c|c|c|c|c|}
\hline parameter & $r$ & $P$ & parameter & $r$ & $P$ \\
\hline Abstinence days & $0 \otimes 05$ & OQ 41 & $\mathrm{PR} \%$ & $\nabla 0 \otimes 49$ & 0.002 \\
\hline Age & OQ 15 & 0ष 07 & sperm survival rate & $\nabla 0 \otimes 56$ & 0.002 \\
\hline Sperm concentration & $\triangle 0 \otimes 03$ & O\ 81 & $\mathrm{Zn}$ & 0.15 & 0.20 \\
\hline Semen volume & $0 \otimes 03$ & OQ 51 & Fru & -0.20 & 0.05 \\
\hline Percentage of normal sperm & -0.16 & 0.06 & a-Glu & -0.03 & 0.82 \\
\hline
\end{tabular}

Sperm DFI was negatively correlated with sperm survival rate and PR\% ( $r=-0.56 .-0.46$, respectively, $P<0$. $01)$, There was no correlation with age, abstinence days, semen volume, sperm concentration, percentage of normal sperm, Fru, a-Glu, Zn ( $r=0.15,0.05,0.03,-0.03,-0.20,-0.16,-0.20,-0.03,0.15, p>0.05)$.see Table 3.

MDA content and TAC levels were compared between the two groups

Table 4 MDA content and TAC levels were compared between the two groups 


\begin{tabular}{|llll|}
\hline Group & number of cases & MDA (nmol/ml) & TAC(U/L) \\
\hline High DFI & 90 & $9.60 \pm 2.03^{*}$ & $10.21 \pm 2.16^{*}$ \\
\hline Low DFI & 61 & $5.12 \pm 1.80$ & $20.35 \pm 2.56$ \\
\hline$t$ & 6.01 & 11.65 \\
\hline$P$ & $<0.05$ & $<0.05$ \\
\hline
\end{tabular}

Compared with Group Low DFI, *: $P<0.05$.

DFI was used as grouping basis, DFI $30 \%$ was low DFI group (61 cases), and DFI $\geq 30 \%$ was high DFI group (90 cases). There was no statistically significant difference in general data between the two groups $(P>0.05)$, indicating comparability. MDA content in high DFI group was higher than that in low DFI group. TAC levels were lower than those in the low DFI group, The difference was statistically significant $(P<0.05)$. See Table 4.

\section{Correlation analysis between sperm DNA integrity and sperm oxidative stress index}

Table 5 Correlation analysis between sperm DNA integrity and sperm oxidative stress index

\begin{tabular}{|ll|}
\hline Group & DFI \\
\hline MDA & 0.42 \\
\hline TAC & -0.40 \\
\hline
\end{tabular}

Pearson correlation analysis showed that DFI was positively correlated with MDA content $(r=0.42$, $P<0.01)$ and negatively correlated with $\operatorname{TAC}(r=-0.40, P<0.01)$. See Table 5.

\section{Discussion And Conclusions}

SDF was produced in sperm cells during spermatogenesis and maturation periods, which can reflect the damage status of chromosome and integrity of impaired DNA ${ }^{[12-13]}$. The SDF index (DFI) is used to assess the degree of sperm DNA impairment. Damage on sperm chromatin can directly affect the sperm's normal physiological functions ${ }^{[14]}$, and also leads to transmit the incorrect genetic information to the offspring. Currently, three factors are considered to be the main cause of sperm damage, including abnormal sperm chromatin assemble, aberrant sperm cell apoptosis, and excessive oxidative stress ${ }^{[15]}$. During sperm maturation, histones are gradually replaced by the arginine-rich, cysteine-rich and smaller protamine (HP), and this process reduces the self-repair ability of sperm DNA in response to internal changes and external environment. Furthermore, under the effect of torsion-tension that is generated by 
the double-stranded DNA helix, the misfolding of DNA supercoiling structures in the chromosome can also lead to aberrant DNA repair, which result in abnormalities of the chromatin structures abnormalities and SDF increase ${ }^{[16]}$. In addition, inflammation in the external genital tract and varicocele can also increase the production of SDF by inducing free radical-mediated oxidative stress in the sperm ${ }^{[17]}$.

Many studies have proposed hypotheses on the mechanism of sperm DNA damage. The main possible reasons are as follows: 1) Antioxidants in semen can protect the oxidative damage of sperm DNA, and a small amount of appropriate ROS can help sperm capacitation and acrosomal reaction. Excessive ROS removal ability and defensive ability, more than itself lead to sperm DNA double chain rupture, produce single, its principle is ROS may through direct oxidation of sperm DNA bases, may through covalent binding of lipid peroxidation products and DNA, causing the sperm DNA chain rupture, leading to a biological structure of sperm, sperm DNA damage 2)Abnormal sperm chromatin assembly can lead to sperm DNA double strand break. The main link leading to sperm nuclear DNA damage is the abnormal appearance of protosomal proteins when they replace histones ${ }^{[18-22]}$. During the sperm formation process, nucleocapsid proteins of chromatin undergo the transformation from histones to transition proteins to protosomal proteins to concentrate chromatin and maintain the normal morphology and DNA integrity of sperm. In damaged or immature sperm, a large amount of sulfhydryl (SH) of protamine cannot be oxidized into disulfide bonds, so it cannot bind closely with DNA. As a result, loose chromatin is formed and its structure is unstable. Its DNA is denatured into single strand under the action of acid, which eventually leads to sperm DNA damage. Therefore, sperm DFI can be detected to reflect the defect degree of genetic material in sperm nucleus and sperm DNA maturity status.

In this study, The low DFI group had an obviously lower seminal MDA level and an obviously higher seminal TAC level than high DFI groups, indicating that too much MDA was produced during seminal lipid peroxidation and that the drop of TAC level triggered oxidative stress reaction and destroyed the spermatic membranes ${ }^{[21]}$. According to Ni et al. ${ }^{[23]}$ and Fu et al. ${ }^{[24]}$, sperm DNA damage could be caused by ROS in patients with varicocele. Shang et al. ${ }^{[25]}$ and Greco et al. ${ }^{[26]}$ have reported that antioxidant can decrease the rate of DNA fragmentation, suggesting that the seminal ROS participates in the process of sperm DNA damage.

The results of this study showed that sperm DFI level was significantly negatively correlated with sperm survival rate and $\mathrm{PR} \%$, which was consistent with relevant research reports ${ }^{[27-29]}$, and there were statistical differences among groups I, II and III. The survival rate and PR\% of sperm decreased significantly with the increase of DFI level, but this does not mean that the lower percentage of the former means that sperm DNA is damaged. However, damaged sperm DNA can affect sperm fertilization ability and even inhibit embryo development. This study showed that there was a statistically significant difference in Fru content between group II and III and group I, while there was no statistically significant difference between group II and group III. However, most PR\% of these two groups was lower than the lower limit of WHO reference value ${ }^{[30]}$, which was classified as asthenospermia. However, spermatozoa Fru is closely related to sperm motility. Fru provides energy source for sperm motility, and the reduced 
content of Fru can reduce sperm motility ${ }^{[31]}$. The seminal plasma Fru originates from the seminal vesicle and mainly reflects its secretion function. In this study, inflammation and infection have been excluded, which may be related to the endocrine function of the seminal vesicle and other factors. In summary, sperm DFI was low, sperm Fru was high, and sperm PR\% was high. The results also showed that the percentage of normal sperm in group III was statistically different from that in group II, which was consistent with some reports ${ }^{[32]}$, and the former was significantly lower than the latter. Although there was no statistical difference between group I and the other two groups, the percentage of normal sperm was higher than that of group III and lower than that of group II .In this study, no correlation was found between patient age, abstinence days, semen volume, sperm concentration, spermatozoal G-glu and spermatozoal Zn and sperm DFI. However, it can be seen from table 2 that DFI increases with age, and it has been reported that age is positively correlated with DFIQWith the aging of male organs such as testis, prostate and epididymis, it leads to the increase of reactive oxygen species (ROS) and the decline of antioxidant capacity. Too much ROS produces a large amount of lipid peroxides, which attack the sperm cell membrane, causing the sperm DNA strand to break and destroy it integrity ${ }^{[33]}$, which may be due to insufficient sample size in this study. Semen volume and sperm concentration were mainly related to the secretion volume of accessory gonads and total sperm count, but no correlation was found between them and DFI. About $60 \%$ of the seminal plasma is from the seminal vesicle, $30 \%$ from the prostate, and the rest from epididymis, paraurethral gland, paraurethral gland, testis, etc. The spermatic a-Glu and spermatic $\mathrm{Zn}$ are mainly from the secretion of epididymis and prostate. It has been reported that too high free $\mathrm{Zn}$ has toxic effects on sperm, and its oxide inhibits sperm fermentation and oxidation process ${ }^{\text {[34-36]. }}$ It has also been suggested that $\mathrm{Zn}$ can delay cell membrane lipid oxygenation. In this study, the level of spermatic $\mathrm{Zn}$ in each group was basically within the normal reference range, and it was not clear whether it affected sperm quality ${ }^{[37]}$.

In summary. In future studies, the relationship between a-Glu and DFI can be further explored through large sample analysis of normal group and abnormal group based on the content levels of sperm a -Glu and sperm Zn. As an increasingly common technology in clinical testing for reproduction, sperm DFI has proven to be very valuable in male fertility evaluation.In future studies, the sample size should be expanded to allow more accurate conclusions.

\section{Abbreviations}

IVF in vitro fertilization

Fru Fructose

A-glu a-glucosidase

MDA Seminal plasma malondialdehyde

TAC total antioxidant capacity 
Zn zinc levels

VSL straight line veloctity

SH sulfhydryl

\section{Declarations}

\section{Approval and Consent to participate}

The authors are accountable for all aspects of the work in ensuring that questions related to the accuracy or integrity of any part of the work are appropriately investigated and resolved. The study was approved by the Ethics Committee of Women's Hospital of Nanjing Medical University (No. 20180338) and conducted in accordance with the Declaration of Helsinki (as revised in 2013). All the patients provided the written informed consent.

\section{Availability of supporting data}

The datasets used are analyzed are during the current study are available from the corresponding author on reasonable request.

\section{Consent for publication}

Not applicable.

\section{Competing interests}

The authors declare that they have no competing interests.

\section{Funding}

This work was supported in part by 333 high level talents training project of Jiangsu Province and Six talent peaks project in Jiangsu Province (WSN-121).

\section{Author contributions}

K.S. L wrote the main manuscript, F.P prepared all the figures, X.D.M $₫$ X.Y.Y provided assistance with data acquisition. All authors reviewed the manuscript.

\section{Acknowledgments}

We thank Dr X.Y.Y, Department of Obstetrics and Gynecology, the First Affiliated Hospital of Xi'an Jiaotong University, Xi'an, Shannxi 710061, P. R. who took part with great efforts in revising the paper in English. 


\section{References}

1. HO. WHO laboratory manual for the Examination and processing of human semen. Switzerland: WHO Press; 2010.

2. Kobayashi T, Miyazaki T, Natori M, Nozawa S. Protective role of superoxide dismutase in human sperm motility: superoxide dismutase activity and lipid peroxide in human seminal plasma and spermatozoa. Human Reprod. 1991;6:987-991.

3. Lopez G, Lafuente R, Checa MA, Carreras R, Brassesco M. Diagnostic value of sperm DNA fragmentation and sperm high-magnification for predicting outcome of assisted reproduction treatment. Asian J Androl. 2013;15(6):790-794.

4. Guo YL, Xin ZC, Jin J. Male reproductive medicine. Beijing: Peking University Medical Press,2016. 1.

5. World Health Organization. Laboratory manual for Human semen Examination and Treatment. Version 5. Beijing: People's Medical Publishing House, 2011. 192.

6. Xu XR, Lin H, Zhang XX, Li JY, Zhang W, Sun WJ, Pan YM. The effects of extremely low frequency electromagnetic field exposure on the $\mathrm{pH}$ of the adult male semen and the motoricity parameters of spermatozoa in vitro. Zhonghua Lao Dong Wei Sheng Zhi Ye Bing Za Zhi 2012;30:178-180.

7. Liu XC, Chen GS, He XL. Sperm DNA damage is not related to seminal leukocyte subsets in infertile males with asymptomatic genital tract infection. National Journal of Andrology 2018;24:45-9.

8. Enginsu ME, Dumoulin JC, Pieters MH, Bras M, Evers JL, Geraedts J.P.M. Evaluation of human sperm morphology using strict criteria after Diff-Quik staining: correlation of morphology with fertilization in vitro. Hum Reprod 1991;6:854-858.

9. Liu KS, Mao XD, Pan F, Chen YJ. Application of leukocyte subsets and sperm DNA fragment rate in infertile men with asymptomatic infection of genital tract. Ann Palliat Med 2020. doi: 10.21037/apm19-597.

10. World Health Organization. WHO Laboratory Manual for the Examination and Processing of Human Semen. 5th ed. Geneva: World Health Organization 2010; 84-132.

11. Barratt CL, Aitken RJ, Bjorndahl L, et al. Sperm DNA: organization, protection and vulnerability: from basic science to clinical applications-a position report. Hum Reprod 2010;25:824-38.

10.1093/humrep/dep465

12. Cai Wen- wei,Mo Dun- sheng,Jiang Nan,Wang Ling- li. Seminal elastase detection. Association of sperm DNA integrity with seminal plasma oxidative stress and its influence on in vitro fertilization in infertile males. National Journal of Andrology 2016;22: 892-896.

13. Osman A, Alsomait $H$, Seshadri $S$, et al. The effect of sperm DNA fragmentation on live birth rate after IVF or ICSI: a systematic review and meta-analysis. Reprod Biomed Online 2015;30:120-7. 10.1016/j.rbmo.2014.10.018

14. Gomes M, Goncalves A, Rocha E, et al. Effect of in vitro exposure to lead chloride on semen quality and sperm DNA fragmentation. Zygote 2015;23:384-93. 10.1017/S0967199413000671 
15. Sakkas D, Alvarez JG. Sperm DNA fragmentation: mechanisms of origin, impact on reproductive outcome, and analysis. Fertil Steril 2010;93:1027-36. 10.1016/j.fertnstert.2009.10.046

16. Wright C, Milne S, Leeson H. Sperm DNA damage caused by oxidative stress: modifiable clinical, lifestyle and nutritional factors in male infertility. Reprod Biomed Online 2014;28:684-703. 10.1016/j.rbmo.2014.02.004

17. Min W, Jian S, Li W, Gao XH, Lu X, Zheng MW, Wang YW, Liu K, Tao JW, Wu Y. Assessment of density gradient centrifugation (DGC) and sperm chromatin dispersion (SCD) measurements in couples with male factor infertility undergoing ICSI. J Assist Reprod Genet 2014; 31: 1655-1663.

18. Omran HM,Bakhiet M,Dashti MG.DNA integrity is a critical molecular indicator for the assessment of male infertility. Mol Med Rep 2013;7: 1631-1635.

19. Muratori M, Marchiani S, Maggi M, et al. .Origin and biological sig-nificance of DNA fragmentation in human spermatozoa. Front Biosci 2006; 11: 1491-1499.

20. Gomes $M$, Goncalves $A, R o c h a ~ E$,et al.Effect of in vitro exposure to lead chloride on semen quality and sperm DNA fragmentation. Zygote 2015; 23: 384-393.

21. Shamsi MB, Kumar R, Dada R. Evaluation of nuclear DNA damage in humanspermatozoa in men opting for assisted reproduction. Indian J Med Res 2008; 127:115-123.

22. Ni K, Steger $K$, Yang H, Wang H, Hu K, ZhangT, ChenB. A comprehensive investigation of sperm DNA damage and oxidative stress injury in infertile patients with subclinical, normozoospermic, and astheno/oligozoospermic clinical varicocoele. Andrology 2016; 4:816-824.

23. Fu H, Song WK, Ling XH, Gao CF, Chen ZY, Zhang J,Jiang FN.Correlation of oxidative stress with sperm DNA integrity and semen parameters in infertile men with varicocele. Nat $\mathrm{J}$ Androl 2016;22:530-533.

24. Shang XJ, Mo DS, Zhan XX, Cai HC, Ge JP,Huang YF. L-carnitine protects sperm DNA damage in male infertility. J Third Mil Med 2015;37:1508-1512.

25. Greco E, lacobelli M, Rienzi L, Ubaldi F, Ferrero S, Tesarik J. Reduction of the incidence of sperm DNA fragmentation by oral antioxidant treatment. J Androl 2005;26:349-353.

26. Simões R, Feitosa WB, Siqueira AF, et al. Influence of bovine sperm DNA fragmentation and oxidative stress on early embryo in vitro development outcome. Reproduction 2013; 146: 433-441.

27. Gu XL ,LI HG,Xiong CL Correlation of sperm DNA fragmentation index with age and semen parameters in infertile men.National Journal of Andrology,2018,24: 608-612.

28. Zhang NF,Liu CX,Zheng LY,OUS b,Liang HX,Zhong HL,Chen JH.Diagnostic value of DNA fragmentation index and acrosin activity in male infertility. Jounal of sun yat sen university (Medical sciences) 2018, 39: 93-100.

29. Li F,.Yang H,Xiao JY,Wang LM,Huang Y,Wang Y,Teng XM..A preliminary study on the correlation between human sperm DNA fragmentation index and semen parameters. Chinese Journal of Andrology $2018,32: 33-36$. 
30. World Health Organization. Laboratory manual for Human semen Examination and Treatment. Version 5. Beijing: People's Medical Publishing House 2011. 192.

31. Lu JC.Automatic detection and clinical application of semen biochemical markers. National Journal of Andrology 2018,24: 291-296.

32. Ji XZ, Yang J, Song J, et al. Analysis of correlation between sperm DNA integrity and routine semen parameters in 334 infertile males. Journal of Reproductive Medicine 2017,26 : 1233-1237.

33. Lu JC. Related factors of sperm DNA damage: Advances in studies.National Journal of Andrology2015,21: 675-680.

34. Zhang DM, XU YJ, Zeng LW, et al. Analysis of the relationship between seminal plasma Zinc content and semen quality. In J Lab Med 2012,33 : 2167-2168.

35. Liu P Sun JM, Chi LK, Chen N. Correlation analysis of seminal plasma a-glucosidase,zinc and sperm morphologicalparameters in patients with idiopathic oligospermia. In J Lab Med 2017,38 : 21852187.

36. Liu P, Sun JMg, Han WJ, Liang GQ, Mao JM, Chen N.Correlation analysis of sperm DNA fragmentation index with seminal plasma biochemical index and semen parameters. National Journal of Andrology 201925: 272-274. 